\title{
Relación entre Motivación, Locus de Control, Hábitos de Estudio y Rendimiento en Estudiantes Universitarios de la Facultad de Ingeniería Industrial y de Sistemas de la Universidad Nacional de Ingeniería
}

\author{
Relationship Between Motivation, Locus of Control, Studying \\ Habits and Performance in University Students from the \\ Industrial and Systemas Faculty from the National Engineering \\ University
}

\author{
Mario Heinrich Fisfalen Huerta ${ }^{1}$ \\ ${ }^{1}$ Facultad de Ingeniería Industrial y de Sistemas de la Universidad Nacional de Ingeniería, Lima, Perú.
}

\begin{abstract}
RESUMEN
El rendimiento académico de los estudiantes depende de una serie de factores principalmente internos al estudiante; dentro de los cuales los hábitos de estudio juegan un rol muy importante, así como la motivación de los estudiantes y el locus de control. En el presente trabajo, se hace un estudio de la relación entre rendimiento académico, hábitos de estudio, motivación y locus de control; analizando la incidencia de las tres últimas variables sobre la primera. Para lo cual se aplica la técnica de la encuesta así como escalas de motivación, de hábitos de estudio y de locus de control; analizando los resultados con el empleo de técnicas estadísticas como el análisis de correlación, el análisis de varianza y el análisis de correlación. Los resultados del estudio muestran que los hábitos de estudio, así como la motivación hacia la afiliación y la motivación al logro inciden significativamente en el rendimiento académico de la muestra, conformada por estudiantes de la Facultad de Ingeniería Industrial y de Sistemas.
\end{abstract}

Palabras clave.- Rendimiento académico, Hábitos de estudio, Necesidad de logro, Necesidad de filiación, Necesidad de poder, Locus-D control.

\begin{abstract}
The academic performance of students depends on a number of factors, mostly psycological; within which studying habits play an important role, as well as student motivation and locus of control. In this work, a study of the relationship between academic performance, study habits, motivation and locus of control is made; analyzing the impact of the last three variables over the first. For which the survey technique is applied and scales of motivation, study habits and locus of control; analyzing the results with the use of statistical techniques such as correlation analysis, analysis of variance and correlation analysis. The study results show that the study habits and motivation for affiliation and motivation to achieve significant impact on the academic performance of the sample, formed by students of the School of Industrial and Systems Engineering.
\end{abstract}

Key words.- Academic performance, Study habits, Need for achievement, Need for affiliation, Need for power, Control-D locus.

\section{INTRODUCCIÓN}

Mucho se ha discutido sobre los factores asociados con el rendimiento de los estudiantes tal vez la mayor parte de los estudios se han abocado al trabajo con escolares, ósea niños y adolescentes de los niveles básico y secundario. Existen también diversos factores que pueden influir en la misma; sin embargo, nosotros hemos seleccionado los factores motivación y hábitos de estudio. Se ha elegido la teoría de necesidades de McClelland, que presenta las necesidades de logro, de afiliación y de poder, puesto que consideramos que nos provee un instrumento importante para medir algunos de los factores que podrían incidir en el rendimiento académico, como son la motivación de logro, de afiliación y de poder. Así mismo, consideramos que los hábitos de estudio son factores que pueden hacer la diferencia entre el mayor o menor rendimiento en los estudios. Por otro lado, la teoría del locus de control podría explicar 
el esfuerzo que se haga no se omita en mejorar el rendimiento académico puesto se podría esperar que las personas que esperan que lo que logren en la vida se deba a condiciones externas, no se esforzarían por superarse.

\section{ANTECEDENTES}

En el año 2003, Yesica Reyes Tejada, presenta una investigación titulada "Relación entre Rendimiento académico, La ansiedad ante los exámenes, los rasgos de personalidad, el auto concepto y la asertividad en estudiantes del Primer Año de Psicología de la UNMSM", llegando a las siguientes conclusiones:

- El rendimiento académico del grupo estudiado correlaciona significativamente y de manera positiva con el auto concepto académico y el auto concepto familiar, hallándose además una correlación negativa con los rasgos de personalidad sumisión - dominancia (factorE).

- La ecuación de regresión múltiple nos muestra al auto concepto académico como predictor del rendimiento de los estudiantes de primer año de Psicología que fueron estudiados, con una relación fuertemente significativa (REYES TEJADA, 2003).

\section{SOPORTE TEÓRICO DE LAINVESTIGACIÓN}

\section{Motivación}

"Voluntad para hacer un gran esfuerzo por alcanzar las metas de la organización, condicionada por la capacidad del esfuerzo para satisfacer alguna necesidad personal" (VALDES HERRERA, 2006).

Teoría de McCleland.- David McClelland fue un psicólogo estadounidense, conocido por su trabajo sobre la teoría de la necesidad.

Plantea que una vez que el individuo ha logrado satisfacer sus necesidades básicas o primarias, la conducta del individuo pasa a estar dominada por tres tipos de necesidades: necesidades de afiliación, que implican el deseo de mantener relaciones interpersonales amistosas y cercanas; necesidades de logro, que incluyen el impulso de sobresalir, de alcanzar metas, de vencer obstáculos y tener éxito; y necesidades de poder, que implican el deseo de ejercer influencia sobre individuos y situaciones para hacer que ocurran ciertas cosas que de otra forma no ocurrirían. Esta teoría parte de las siguientes premisas:

? El ambiente social y cultural que rodea a los seres humanos a lo largo de la vida va a determinar en buena medida sus necesidades.

? La infancia es un período especial para la vida de cada sujeto. En esta etapa en la que los sujetos aprendemos gran partes de nuestras necesidades.

?. Las vivencias que tenemos determinan que deseemos satisfacer más unas necesidades que otras.

? Las necesidades que los sujetos tienen se resumen en la afiliación, el poder y logro (RODRIGUEZ, pág. $10 ; 11)$.

Lamotivacióndelogroeraconsideradapor McClelland como la principal causa de desarrollo y progreso económico de las sociedades. También estudiaron otros motivos como la afiliación, el poder. Al interior de las teorías de la motivación, la de McCleland es clasificada como una de las teorías hedonistas y de activación. El concepto central de estas teorías es el afecto: lo placentero y lo desagradable. Estas teorías señalan que la conducta es más eficiente con ciertos niveles generales de activación, y que los aumentos de tensión pueden recompensar si el alertamiento (arousal) general es elevado hasta un punto adecuado (Cofer y Appley, 1971). Para McClelland toda motivación se basa en la emoción y consiste, concretamente, en una expectativa de cambio en la condición afectiva. Un motivo es entonces el restablecimiento, por un indicio, de un cambio de una situación afectiva. En esta definición, restablecimiento significa que está involucrado un aprendizaje previo. En el sistema de McClelland, todos los motivos sociales son aprendidos. (PERRILLA TORO, 1998, pág. $529 ; 532)$.

La teoría de McClelland se basa en tres necesidades:

?. Necesidades de Realización, su interés es desarrollarse, destacarse aceptando responsabilidades personales, se distingue además por intentar hacer bien las cosas, tener éxito incluso por encima de los premios. Buscan el enfrentamiento con problemas, desean retroalimentarse para saber sus resultados y afrontan el triunfo o el fracaso.

? Necesidades de Poder, su principal rasgo es el de tener influencia y control sobre los demás y se afanan por esto Prefieren la lucha, la competencia y se preocupan mucho por su prestigio y por influir sobre las otras personas incluso más que por sus resultados.

? Necesidades de Filiación, su rasgo esencial ser solicitados y aceptados por otros, persiguen la amistad y la cooperación en lugar de la lucha, buscan comprensión y buenas relaciones (VALDES HERRERA, 2006). 


\section{Hábitos de estudios}

Paradigma conductista.- Se considera el estudio como una compleja secuencia de acciones elección del tema, organización del material, lectura del texto y adquisición de contenidos.

Lo que se busca es conocer las situaciones antecedentes de la conducta de estudio y utilizar refuerzos apropiados que permitan obtener rendimientos satisfactorios. Algunas de las consideraciones ofrecidas por este paradigma respecto al estudio son:

? Las condiciones del estudio deben ser apropiadas: temperatura, ventilación, mobiliario, iluminación, organización de materiales, tiempo de estudio, horarios, etc.

? Uso de técnicas de estudio: diferenciación entre ideas principales y secundarias, realización de esquemas y resúmenes, utilización de diccionarios, manejo de ficheros, etc.

[? Estructurar la tarea de estudio, dividiéndola en sus componentes específicos y desarrollando el aprendizaje gradualmente.

?. Tener en cuenta la curva de aprendizaje: precalentamiento, ascenso, meseta, descenso y fatiga.

? Incluir períodos de descanso que permitan disminuir el cansancio y potenciar el aprendizaje.

? ? Potenciar la motivación del alumno por elestudio.

? Utilizar refuerzos que faciliten y consoliden los hábitos y técnicas de estudio

Paradigma cognitivo.- Se centra sobre todo en el aprendizaje de contenidos $\mathrm{y}$, por tanto, enfatiza la importancia de la memoria, la codificación y la recuperación informativa. Las principales aportaciones son:

? Hay un interés especial por las estructuras y procesos cognitivos más relevantes: almacenes informativos, representaciones mentales, relación entre informaciones viejas y nuevas, leyes del olvido, etc.

2. La mente es un "sistema constructor" de la información.

3. La estructura del texto se corresponde con las estructuras mentales. De acuerdo con este principio, el texto es un medio extraordinario para comprender el funcionamiento de la mente humana y la actividad de estudiar.

? Para mejorar el estudio es necesario desarrollar ciertas operaciones cognitivas.

? Las técnicas de trabajo intelectual adquieren especial importancia en el estudio, porque permiten entrenar al alumno en determinadas estrategias de atención, elaboración y organización de la información, al tiempo que se favorece la meta cognición o regulación de los propios procesos de pensamiento y de aprendizaje.

Perspectiva humanista.- De acuerdo a la perspectiva humanista, cualquier plan de adquisición de hábitos de estudio debe partir de las necesidades, capacidades, afectos, actitudes y valores de los alumnos, sin que ello nos haga olvidar el papel de la orientación (MARTINEZ OTERO, 2005, pág. 1;8).

\section{Locus de control}

La definición de locus de control fue establecida por Rotter (1966) considerando que "si la persona percibe que el acontecimiento es contingente con su conductao sus propias características relativamente permanentes, se ha dicho que es una creencia en el control interno"; en cambio, sostiene que "cuando un refuerzo es percibido como siguiendo alguna acción personal, pero no siendo enteramente contingente con ella, es típicamente percibido, en nuestra cultura, como el resultado de la suerte (...), y en este sentido se ha dicho que es una creencia en el control externo" (p. 1). Siguiendo tal diferenciación, Rotter (1966) elabora una escala bidimensional de internalidad-externalidad de locus de control en sujetos adultos con 29 ítems, cada ítem con dos enunciados generales sobre las causas genéricas del éxito y el fracaso en la vida en general, o en particular (en los estudios, el trabajo, las relaciones interpersonales, etc.) (VISDOMINE OTERO, 2006, pág. 729;751).

\section{Rendimiento académico}

El rendimiento académico es, según Pizarro (1985), una medida de las capacidades respondientes o indicativas que manifiesta, en forma estimativa, lo que una persona ha aprendido como consecuencia de un proceso de instrucción o formación; desde la perspectiva del alumno, define al rendimiento académico como la capacidad respondiente de éste frente a estímulos educativos, la cual es susceptible de ser interpretada según objetivos o propósitos educativos ya establecidos. Tenemos también que en 1985, Himmel (cit. por Castejón Costa, 1998) define el rendimiento académico o efectividad escolar como el grado de logro de los objetivos establecidos en los programas oficiales de estudio. Por otro lado, el rendimiento académico, para Novaez (1986), es el quantum obtenido por el individuo en determinada actividad académica. Así, el concepto del rendimiento está ligado al de aptitud, y sería el resultado de ésta y de factores volitivos, afectivos y emocionales, que son características internas del sujeto. 
El rendimiento académico es multidimensional pues en él inciden multitud de variables (Gimeno Sacristán, 1977). (REYES TEJADA Y. N., 2003, pág. 13;16).

\section{ASPECTOS METODOLÓGICOS}

Tipo y nivel de investigación

La Investigación por su nivel es exploratoria.

Método y diseño de investigación

La investigación por su diseño es no experimental.

Población y muestra

Descripción de la población.- La población está conformada por estudiantes universitarios de la Universidad Nacional de Ingeniería, Facultad de
Ingeniería Industrial y de Sistemas.

Tamaño de la muestra.- El tamaño de la muestra será de 200 individuos.

Tipo de muestra.- Se realizara un muestreo no probabilístico.

Variables.-

a) Motivación:

b) Locus de control

c) hábitos de estudio

d) Rendimiento académico

\section{TÉCNICAS INSTRUMENTOS DE} RECOLECCIÓN DE DATOS

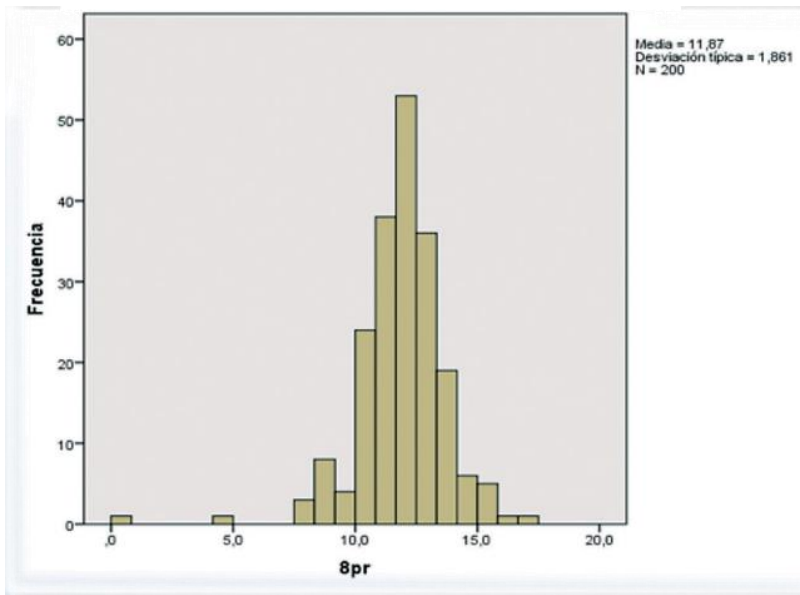

Figura 1. Distribución de frecuencias del promedio ponderado de notas

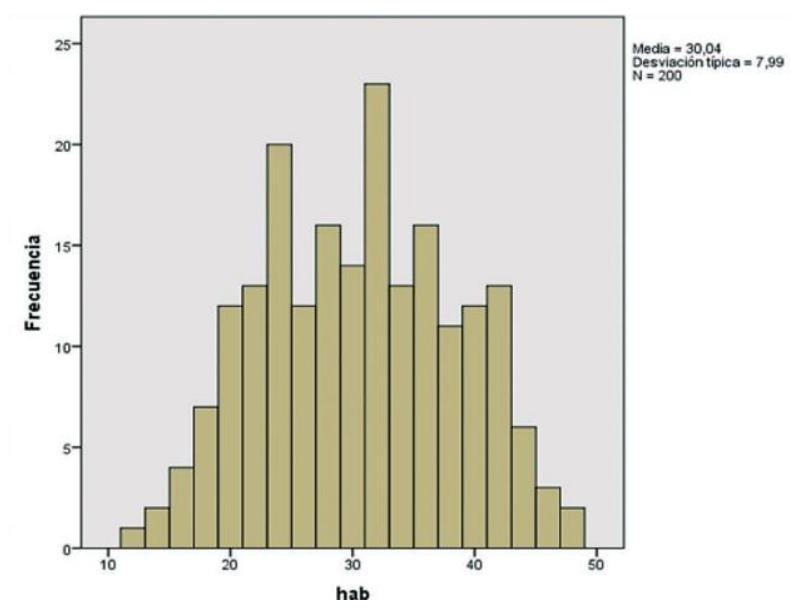

Figura 2. Distribución de frecuencias de hábitos de estudio (elaboración propia). 


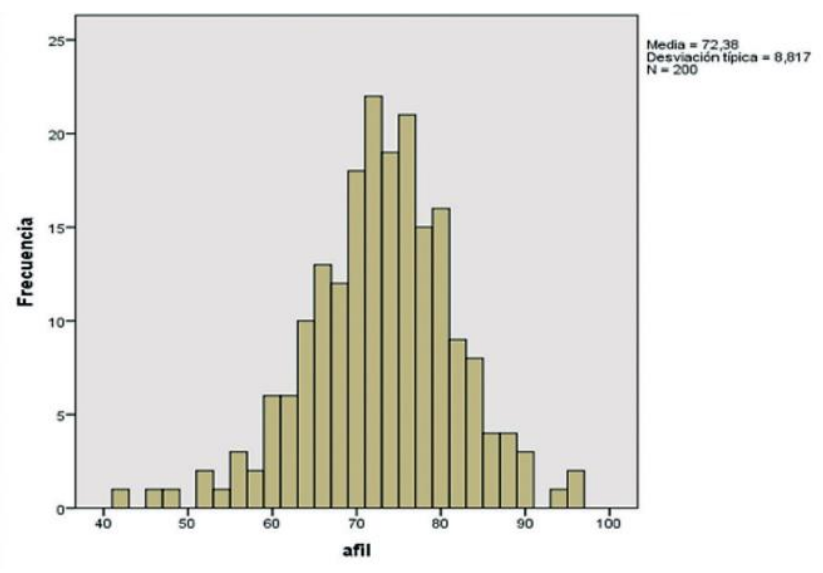

Figura 3. Distribución de frecuencia de motivación d afiliación (fuente y elaboración propia).

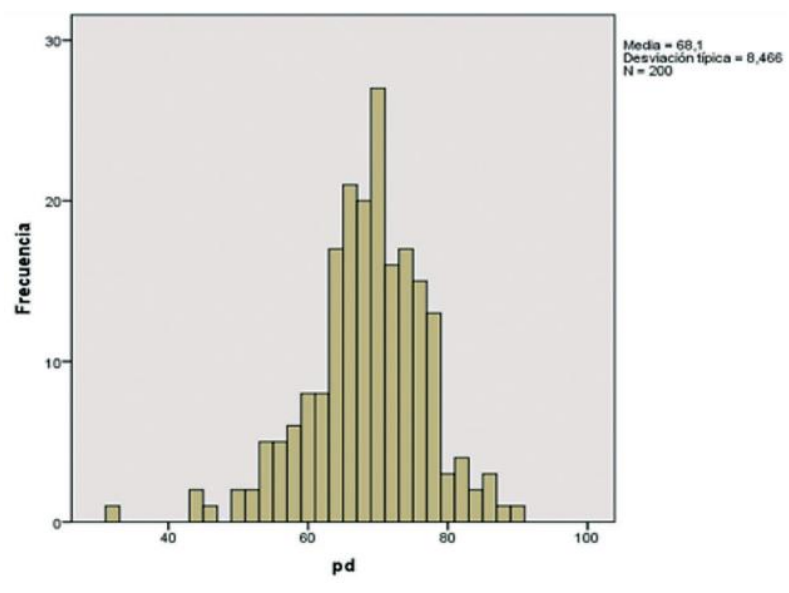

Figura 4. Distribución de frecuencia de motivación de poder (fuente y elaboración propia).

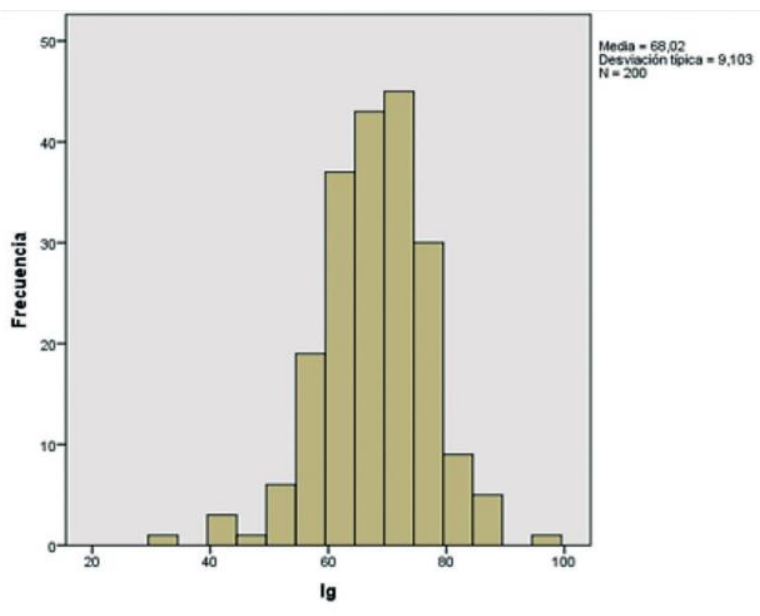

Figura 5. Distribución de frecuencia de motivación de logro (fuente y elaboración propia). 


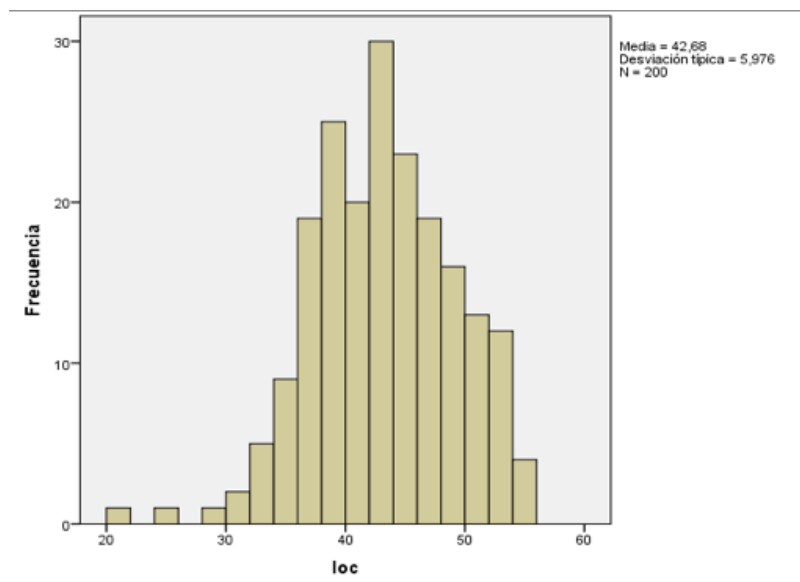

Figura 6. Distribución de frecuencia de locus de control( fuente y elaboración propia)

Tabla 1. Correlación entre variables.

\begin{tabular}{|c|c|c|c|c|c|c|c|}
\hline \multicolumn{8}{|c|}{ Correlaciones } \\
\hline & & 8pr & Hab & Afil & pd & $\lg$ & loc \\
\hline \multirow{4}{*}{$8 \mathrm{pr}$} & Correlación de Pearson & 1 & $197^{* *}$ &, $165^{*}$ & 101 & $144^{*}$ &,- 036 \\
\hline & Sig. (bilateral) & & ,005 & 019 & 157 & 043 & 614 \\
\hline & $\mathrm{N}$ & 200 & 200 & 200 & 200 & 200 & 200 \\
\hline & Correlación de Pearson & $197^{* *}$ & 1 & $352^{* *}$ &, $303^{* *}$ & $195^{* *}$ &, $242^{* *}$ \\
\hline \multirow[t]{3}{*}{$\mathrm{Hab}$} & Sig. (bilateral) & 005 & & ,000 & ,000 & ,006 & 001 \\
\hline & $\mathrm{N}$ & 200 & 200 & 200 & 200 & 200 & 200 \\
\hline & Correlación de Pearson &, $165^{*}$ & $352^{* *}$ & 1 &, $727^{* *}$ &, $576^{* *}$ & ,082 \\
\hline \multirow[t]{3}{*}{ Afil } & Sig. (bilateral) & ,019 &, 000 & & ,000 & 000 & ,249 \\
\hline & $\mathrm{N}$ & 200 & 200 & 200 & 200 & 200 & 200 \\
\hline & Correlación de Pearson & 101 & $303^{* *}$ &, $727^{* *}$ & 1 & ,778** & ,001 \\
\hline \multirow[t]{3}{*}{$\mathrm{Pd}$} & Sig. (bilateral) & 157 & 000 & ,000 & & 000 & ,994 \\
\hline & $\mathrm{N}$ & 200 & 200 & 200 & 200 & 200 & 200 \\
\hline & Correlación de Pearson &, $144^{*}$ &, $195^{* *}$ &, $576^{* *}$ &, $778^{* *}$ & 1 &,- 042 \\
\hline \multirow[t]{3}{*}{ Lg } & Sig. (bilateral) & 043 & ,006 & ,000 & ,000 & & ,553 \\
\hline & $\mathrm{N}$ & 200 & 200 & 200 & 200 & 200 & 200 \\
\hline & Correlación de Pearson &,- 036 & ,242** & ,082 & ,001 &,- 042 & 1 \\
\hline \multirow[t]{2}{*}{ Loc } & Sig. (bilateral) & 614 & 001 & ,249 & ,994 &, 553 & \\
\hline & $\mathrm{N}$ & 200 & 200 & 200 & 200 & 200 & 200 \\
\hline
\end{tabular}

\section{**. La correlación es significativa al nivel 0,01 (bilateral).}

La correlación es significante al nivel 0,05 (bilateral)

Fuente y elaboración propia

Tabla 2. Modelo ANOVA*.

\begin{tabular}{|c|c|c|c|c|c|c|}
\hline & Modelo & $\begin{array}{c}\text { Suma de } \\
\text { cuadrados }\end{array}$ & gl & $\begin{array}{c}\text { Media } \\
\text { cuadrática }\end{array}$ & $\mathbf{F}$ & Sig. \\
\hline \multirow{3}{*}{1} & Regresión & 36,438 & 3 & 12,146 & 3,646 &, $014^{\natural}$ \\
\hline & Residual & 652,872 & 196 & 3,331 & & \\
\hline & & 689,310 & 190 & & & \\
\hline
\end{tabular}

\footnotetext{
* Fuente y elaboración propia

a. Variable dependiente: $8 \mathrm{pr}$

${ }^{\mathrm{b}}$.Variables predictoras: (constante), lg, hab, afil
} 


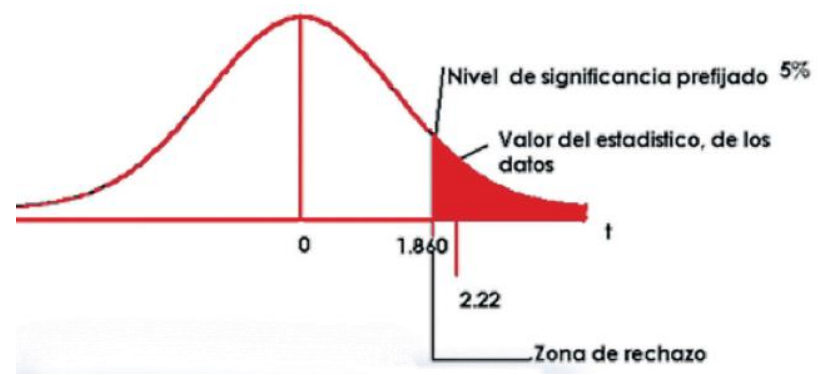

Figura 7. Campana de Gauss.

Tabla 3. Modelo de regresión

\begin{tabular}{|c|c|c|c|c|c|}
\hline \multicolumn{6}{|c|}{ Coeficientes $^{\mathrm{a}}$} \\
\hline \multirow[t]{2}{*}{ Modelo } & \multicolumn{2}{|c|}{ Coeficientes no estandarizados } & $\begin{array}{c}\text { Coeficientes } \\
\text { tipificados }\end{array}$ & $\mathrm{t}$ & Sig. \\
\hline & B & Error típ. & Beta & & \\
\hline (Constante) & 8,708 & 1,161 & & 7,498 & ,000 \\
\hline $\mathrm{Hab}$ & 037 & ,017 & 159 & 2,136 & ,034 \\
\hline Afil & 014 & ,019 & 067 &, 747 & ,456 \\
\hline Lg & ,015 & ,017 & ,074 & ,873 & ,384 \\
\hline
\end{tabular}

Tipos de técnicas e instrumentos a utilizar

Inventario de Hábitos de Estudio CASM-85 Revisión 98

- Escala M-L-1986 Luis A. Vicuña Peri

- Test de locus de control

- Registro de notas y cuestionario de datos personales de los alumnos.

\section{DESCRIPCIÓN DE INSTRUMENTOS A UTILIZAR}

Inventario de hábitos de estudio CASM -85.- Esta prueba forma parte de una Batería de Orientación Vocacional diseñada y creada por el Psic' Luis A. Vicuña Peri con criterios aplicables a nuestra realidad nacional'. El inventario de Hábitos de Estudio CASM - 85, es un test elaborado en nuestro país en el año de 1985 por el Psic. Luis A. Vicuña Peri cuando laboraba en el Colegio de Aplicación de San Marcos (CASM), el propósito de, esta prueba es identificar en el estudiante evaluado los hábitos adecuados e inadecuados al momento de estudiar, motivo por el cual está constituido por un listado de complejos conductuales manifiestos en el momento de estudiar; obtenidos de una relación de 120 conductas que suelen ser comunicadas por los estudiantes que cursan el nivel secundario incluyendo a estudiantes universitarios, y que desde su punto de vista son motivos de su éxito o fracaso académico'. (VICUÑA PARI, 1999, pág. 2:4).

Escala M-L-1986 .- Escala de Motivación M-L 1996, de Luis Alberto Vicuña Peri y cols. Esta escala fue desarrollada en Lima por Luis A. Vicuña, Héctor Hernández y Rune Arauco. Explora la motivación de logro, afiliación y poder en jóvenes y adultos, basándose en la teoría sobre la motivación de Mc Clelland. Posee una validez de 0.01 según la opinión de jueces y una confiabilidad de 0.88 habiéndose utilizado el método de test retest (Tirado Bocanegra, 2007, pág. 24).

Test de Locus de control.- El test se basa en la Teoría de locus de Control, creado por Rotter en 1966.

\section{RESULTADOS}

\section{Análisis descriptivo}

Se aprecia que la distribucióndel promedio ponderado de notas guarda cierta simetría, aproximándose a la distribución de Gauss, Figura 1. La media es de 11.9, la curva es poco dispersa. 
La distribución de frecuencias de los habitos de estudio es un tanto aplanada no pudiéndose definir con claridad un punto central. Hay bastante dispersión, Figura 3.

Esta distribución de frecuencias es concentrada al centro con dos picos, se aprecia una mayor concentración hacia la derecha, hacia puntajes más altos lo que indica una mayor tendencia hacia mayor afiliación, Figura 4.

La distribución de frecuencias de la motivación al poder tiene una forma un tanto irregular: no hay una clara tendencia hacia puntajes altos o majos, Figura 5.

La distribución de frecuencias de motivación al logro es bastante concentrado al centro.

El locus de control muestra una distribución de frecuencias con dos picos, Figura 6.

\section{Análisis correlacional}

Se aprecia que la correlación entre las variables rendimiento académico operacionalizado por promedio ponderado de notas y hábitos de estudio es significativo, con un nivel de significancia del 99.5\%; igualmente son significativas el rendimiento académico con las variables motivación para la afiliación y motivación hacia el logro, Tabla 1.

Lo que nos indica que tanto los que están motivados para el logro como la superación como los motivados a la afiliación tienen mejores resultados, esto se puede explicar por los mejores resultados del trabajo en equipo.

Estadística inferencial.- Análisis de varianza y análisis de regresión, Tabla 2 y 3.

El modelo ANOVA resulta significativo, con una probabilidad de rechazo de solo $1.4 \%$, lo que significa que la mayor variabilidad es explicada porla regresión.

En cuanto al modelo de regresión, se observa que solo el coeficiente de hábitos de estudio es significativo, explicando el rendimiento académico.

Contrastación de hipótesis

La hipótesis principal dice lo siguiente:

"Determinar en qué medida los hábitos de estudio, la motivación y el locus de control inciden sobre el rendimiento académico de los estudiantes de ingeniería industrial y de sistemas de la UNI".

Para contrastar dicha hipótesis vamos a utilizar el modelo de regresión de mínimos cuadrados que establece la relación entre una variable dependiente y varias variables explicativas. Debemos probar que el modelo es válido, con un F significativo, para esto debemos utilizar la prueba F. Y además, que los coeficientes de las variables independientes son significativos, esto lo demostraremos con la prueba t .student. Probaremos que Ho $\neq 0$ lo que significa que la hipótesis nula, es diferente de cero, lo que significa que no podemos aceptar que no hay ninguna relación. Para esto debemos determinar el Nivel de significancia $\leq 5 \%$, que obtenemos de las tablas, lo que significa que solo hay $5 \%$ de probabilidad de equivocarnos

Para el Análisis de correlación, se aprecia la correlación de la variable rendimiento académico con las siguientes variables, Figura 7.

- Hábitos de estudio: Nivel de significancia 0.005 < 0.05 es significativo

- Motivación de afiliación Nivel de significancia $0.019<0.05$ es significativo

- Motivación de logro: Nivel de significancia $0.043<$ 0.05 es significativo

Se demuestra que esas variables correlacionan con rendimiento académico.

Para el ANOVA se tiene un $\mathrm{F}=3.646$ con nivel de significancia $0.014<0.05$; lo que significa que este es significativo, demostrándose que el modelo es viable, por otro lado en el análisis de regresión tenemos el coeficiente de la variable hábitos de estudio, es de 0.159 con un nivel de significancia de $0.034<0.05$ con lo que se demuestra que es significativa y los hábitos de estudios explican la viabilidad en el rendimientoacadémico.

\section{Hipótesis especificas}

a) Determinar en qué medida existe una relación entre hábitos de estudio y rendimiento en estudiantes universitarios de la Facultad de Ingeniería Industrial y del Sistemas de la Universidad Nacional de Ingeniería.

b) Determinar en qué medida existe una relación entre motivación, y rendimiento en estudiantes universitarios de la Facultad de Ingeniería Industrial y del Sistemas de la Universidad Nacional de Ingeniería.

c) Determinar en qué medida existe una relación entre locus decontroly rendimiento en estudiantes 
universitarios de la Facultad de Ingeniería Industrial y del Sistemas de la Universidad Nacional de Ingeniería.

\section{DISCUSIÓN DE RESULTADOS}

En cuanto al análisis descriptivo de las variables, el rendimiento académico, operacionalizado por el promedio ponderado de notas del ciclo anterior, muestra una distribución bastante definida, donde el mayor porcentaje de notas cae en el rango entre $10 \mathrm{y}$ 15 puntos de nota.

La mayor parte de las personas tienen una edad entre 18 y 22 años aunque algunos pueden tener hasta cerca de 30 años. La mayor parte de los encuestados tiene un locus de control relativamente bajo, con un puntaje que es inferior al puntaje medio. La motivación al logro es relativamente alta en la muestra tomada, con una media de 68 sobre 100.

La motivación hacia el poder es también relativamente alto, con una media de 68 y estando la mayoría entre 60 y 80 .

La motivación hacia la afiliación es también relativamente alta, estando la media en 72 , pero es bastante dispersa.

En cuanto a los hábitos de estudio, el puntaje es relativamente bajo, con una media de 30 y con valores bastante dispersos entre 10 y 50 puntos. Esto tal vez se podría explicar por la relativamente alta exigencia de la escala de hábitos de estudio.

\section{Sobre la hipótesis principal}

Si bien se encuentra que las variables independientes inciden sobre el rendimiento académico hay algunas que inciden más que otras y algunas con incidencia significativa.

Así tenemos que la variable hábitos de estudio es la que más incide en el rendimiento académico, seguida por motivación a afiliación y motivación al logro; cada uno incide en forma individual, pero como modelo solo es significativa la variable hábitos de estudio. Esto a pesar que el puntaje promedio de hábitos de estudio es relativamente bajo. Podemos decir que esos pocos hábitos de estudio son suficientes para explicar la variación en el rendimiento académico.

Podemos suponer que las variables motivación por afiliación y motivación por logro individualmente están correlacionados con rendimiento académico, en conjunto su incidencia es menor que el de la variable hábitos de estudio.

\section{Sobre las hipótesis específicos}

Las hipótesis específicas plantean las correlaciones individuales de las variables hábitos de estudio, motivación hacia la afiliación y motivación hacia el logro con la variable rendimiento académico, operacionalizada por el promedio ponderado de notas del ciclo anterior.

Se aprecia que las variables motivación hacia el poder y locus de control no tienen una correlación significativa, siendo que la variable motivación hacia el poder tiene un alto valor mientras que locus de control es bajo.

Esto puede deberse a que la motivación hacia el poder no está directamente relacionado con el rendimiento académico. Así mismo el locus de control alto o bajo, al parecer, no llevar a mejorar el rendimiento académico.

Los buenos hábitos d estudio son los factores que más se relacionan con el rendimiento académico, aunque su nivel puede elevarse mas

Se entiende fácilmente que la motivación hacia el logro pueda estar relacionada con el rendimiento académico

La correlación entre la variable motivación hacia la afiliación y rendimiento académico puede explicarse por la mejora en el rendimiento del trabajo en grupo y por la necesidad de reconocimiento social que incide en el mayor esfuerzo académico.

\section{CONCLUSIONES}

Las variables hábitos de estudio, motivación hacia el logro y motivación hacia la afiliación están individualmente correlacionadas con la variable rendimiento académico, operacionalizada por la variable promedio ponderado de notas del ciclo anterior.

El modelo en conjunto de las variables motivación al logro, motivación a afiliación y hábitos de estudios como variables explicativas de la variable rendimiento académico, es válido como modelo pero solo la variable hábitos de estudio tiene un coeficiente significativa es su relación lineal.

La distribución de frecuencias de la variable hábitos de estudio muestra valores relativamente bajos, lo que indica que los estudiantes podrían mejorar su rendimiento. 
Ladistribucióndefrecuenciasdelavariablemotivación hacia el logro, motivación al poder y motivación a la afiliación muestra valores relativamente altos. Se puede decir que los estudiantes encuestados tienen gran motivación por superarse y por relacionarse en grupo. Habría que evaluar también si la motivación hacia la afiliación en estudiantes de la Universidad Nacional de Ingeniería es acorde con sus habilidades sociales e inteligencia emocional. La distribución de frecuencias del variable locus de control muestra valores bajos.

\section{REFERENCIAS}

[1] Reyes Y 2003 Relación entre Rendimiento académico, la ansiedad ante los exámenes, los rasgos de personalidad el auto concepto y la asertividad en estudiantes del primer año de psicología de la UNMSM UNMSM:Lima

[2] Valdés C 2006 La Motivación [Online] http://www. universidadperu.com/articulo-la-motivacion-universidadperu.php (2014, August)

[3] Rodríguez M J La motivación en el mundo del trabajo [Online] (2015, July)

[4] Perrilla Toro C 1998 Revista Latinoamericana de Psicología 30(3)

[5] Martinez Otero V 2005 Análisis de los hábitos de estudio en una muestra de alumnos universitarios Revista Iberoamericana de Educación

[6] Visdomine Otero C 2006 Locus de control y autorregulación conductual: revisiones conceptual y experimental International. International Journal of Clínical and Health Psychology (3)

[7] Araujo C Una Propuesta de Presentación del Tema de Correlación Simple [Online] https://goo.gl/Qr347t (2015, July)

[8] Motivación Laboral. (s.f.). MOTIVACION LABORAL. [Online] http://motivacionlaboral.galeon.com/teorias.htm (Agosto, 2014)

[9] Orellana L 2001 "Estadística Descriptiva" [Online] http:// www.hacienda.go.cr/cifh/sidovih/cursos/material_de_ apoyo-f-c-cifh/1materialdeapoyocursoscifh/

[10] Pérez de Vargas A 1996 Análisis de la varianza Ed. Centro de Estudios Ramón Areces.

\section{ANEXOS}

\section{CUESTIONARIO DE DATOS PERSONALES Y RENDIMIENTO ACADÉMICO}

1. Lugar de Nacimiento

2. Sexo

3. Edad

4. Ciclo

5. Especialidad I1 I2

6. Curso

7. Sección_

8. Promedio ponderado notas ciclo anterior

9. Puntaje de ingreso a la UNI

10. Nota más baja ex parcial _

INVENTARIO DE HÁBITOS DE ESTUDIO

\begin{tabular}{|c|c|c|c|c|}
\hline 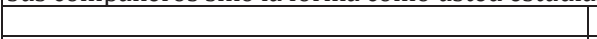 & \begin{tabular}{l|l|} 
Siempre & Nunca \\
\end{tabular} & & Siempre & Nunca \\
\hline I_CÓMO ESTUDIA USTED? & & 29 Confío en que mi amigo me sople. & & \\
\hline $\begin{array}{l}1 \text { Leo todo lo que tengo que estudiar subrayando } \\
\text { los puntos mas importantes. }\end{array}$ & & $\begin{array}{l}30 \text { Confío en mi buena suerte, estudio solo lo que } \\
\text { pienso preguntarán. }\end{array}$ & & \\
\hline
\end{tabular}




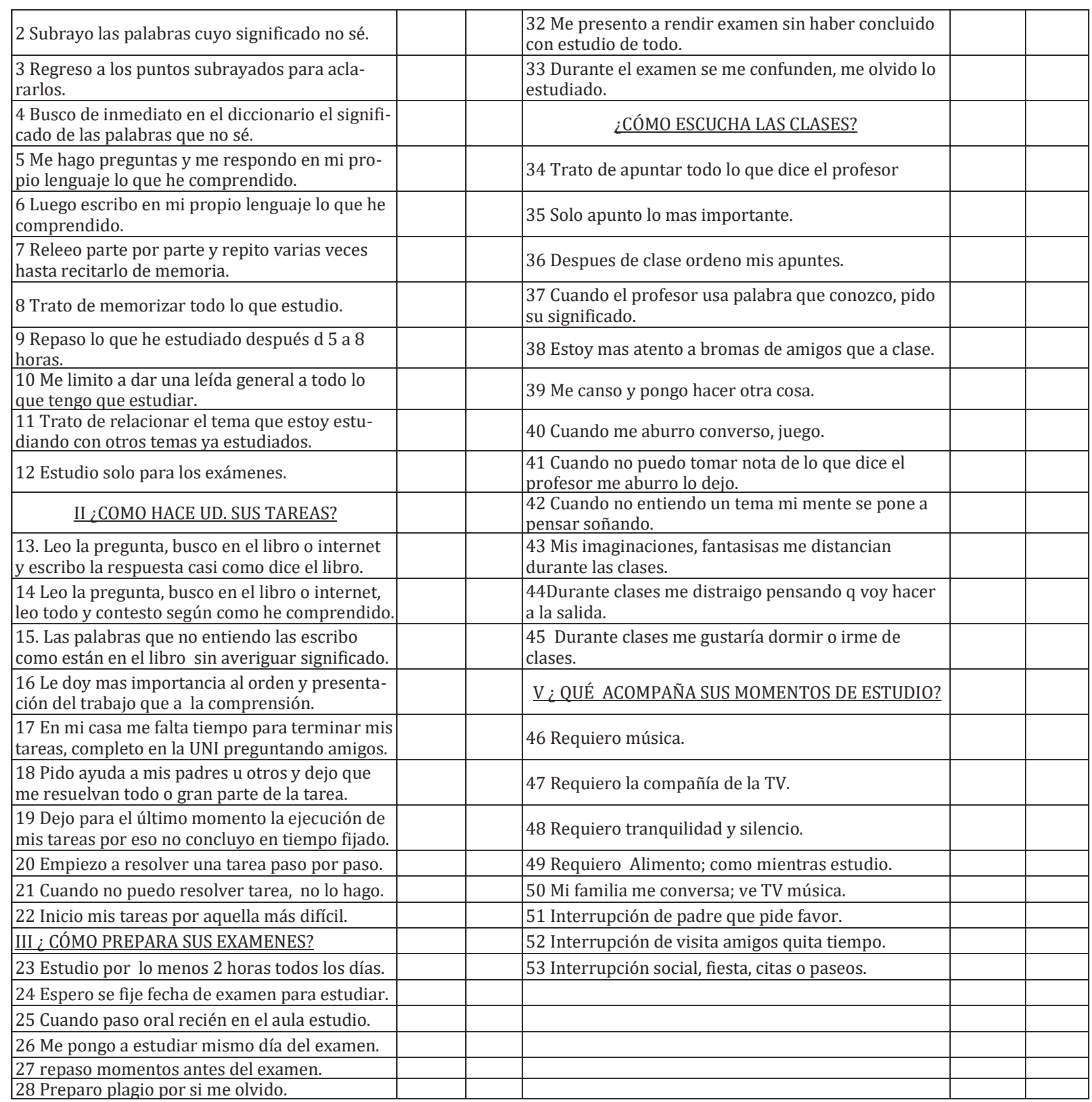

\section{ESCALA M-L 1996}

En cada situación debe indicar su grado de acuerdo o desacuerdo, poner X

1Definitivament desacuerdo 2 Muy en desacuerdo 3 En desacuerdo 4 De acuerdo 5 Muy de acuerdo 6definitivament deacuerdo

\begin{tabular}{|c|c|c|c|c|c|c|c|c|c|c|c|c|c|}
\hline & 1 & 2 & 3 & 4 & 5 & 6 & & 1 & 2 & 3 & 4 & 5 & 6 \\
\hline 1 Cuando estoy con mis padres & & & & & & & 10 con el sexo opuesto & & & & & & \\
\hline A Hago lo necesario por comprenderlos & & & & & & & A Busco puntos de coincidencia. & & & & & & \\
\hline $\begin{array}{l}\text { B Cuestiono lo que me parece inapropia- } \\
\text { do. }\end{array}$ & & & & & & & B Busco controlar la situación. & & & & & & \\
\hline $\begin{array}{l}\text { C Hago lo necesario para conseguir mi } \\
\text { deseo. }\end{array}$ & & & & & & & C Soy simpático si me interesa. & & & & & & \\
\hline 2 En casa. & & & & & & & & & & & & & \\
\hline $\begin{array}{l}\text { A Apoyo en la consecución de algún } \\
\text { objetivo }\end{array}$ & & & & & & & 11 El sexo opuesto & & & & & & \\
\hline B Soy el que da forma a las ideas & & & & & & & $\begin{array}{l}\text { A Es el medio para consolidar mi iden- } \\
\text { tidad sexual }\end{array}$ & & & & & & \\
\hline
\end{tabular}




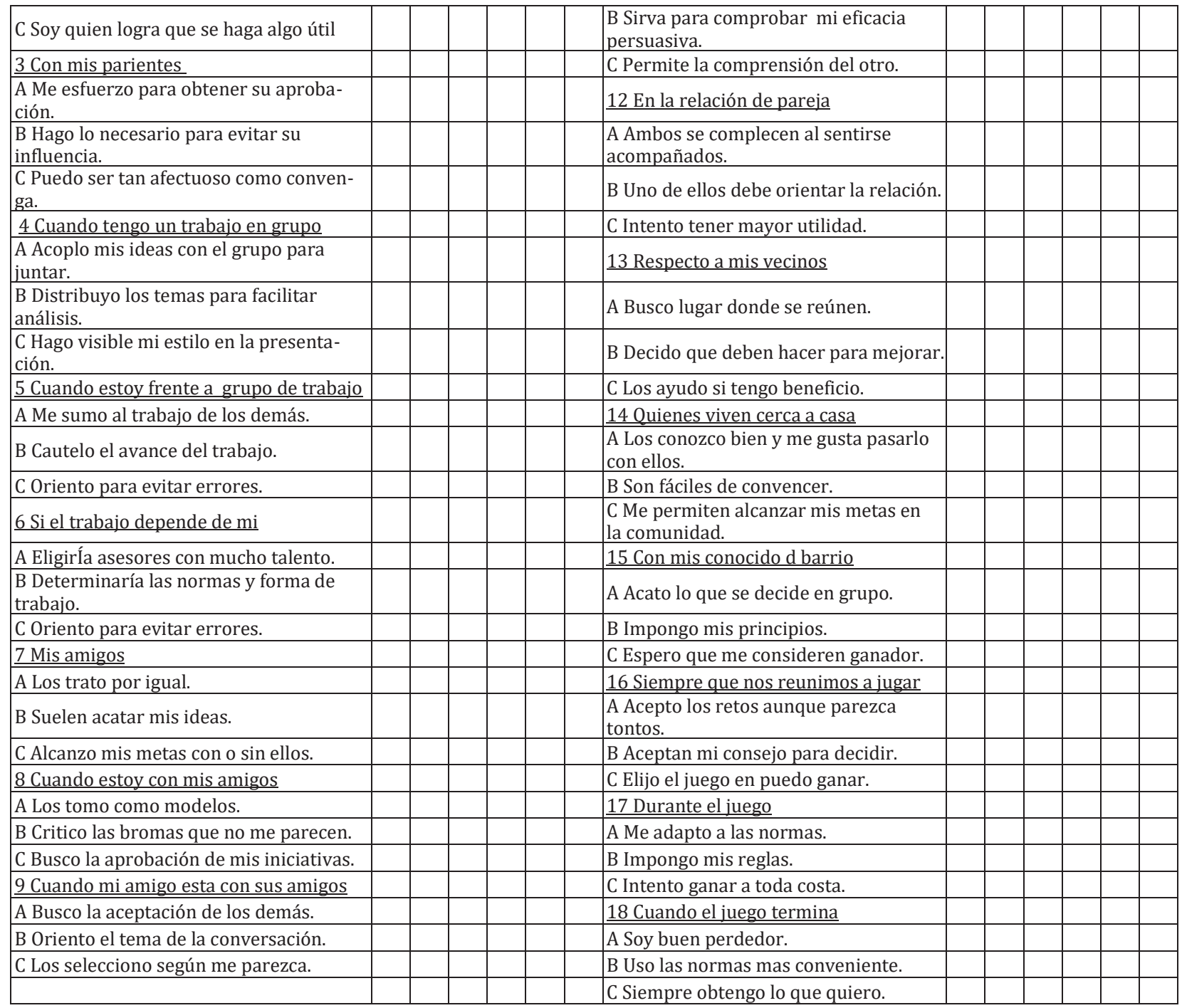

\section{TEST DE LOCUS DE CONTROL}

En cada situación debe indicar su grado de acuerdo o desacuerdo, poner X

1. Definitivamente endesacuerdo

2. Muy en desacuerdo

3. En desacuerdo

4. De acuerdo

5. Muy de acuerdo

6. definitivament de acuerdo

\begin{tabular}{|c|c|c|c|c|c|c|}
\hline & 1 & 2 & 3 & 4 & 5 & 6 \\
\hline \multicolumn{7}{|l|}{1 La herencia determina l a personalidad. } \\
\hline \multicolumn{7}{|l|}{2 El éxito depende de la suerte. } \\
\hline \multicolumn{7}{|l|}{3 No importa lo q planee algo se interpone. } \\
\hline \multicolumn{7}{|l|}{4 En el lugar correcto y en el tiempo correcto es esencial para el éxito. } \\
\hline \multicolumn{7}{|l|}{5 Se nace inteligente no hay nada q se pueda hacer para desarrollar la inteligencia. } \\
\hline \multicolumn{7}{|l|}{ 6 Si logras hacer exitosamente tu trabajo era porque era muy fácil de hacer. } \\
\hline \multicolumn{7}{|l|}{7 Tu puedes determinar o cambiar tu destino. } \\
\hline \multicolumn{7}{|l|}{8 El éxito en UNI resulta del nivel socioeconómico. } \\
\hline \multicolumn{7}{|l|}{9 Si la gente esta sola es porque no le dieron oportunidad de conocer a otros. } \\
\hline 10 Si buscas metas realistas vas lograr éxito. & & & & & & \\
\hline
\end{tabular}

